\title{
Synergistic tomographic image reconstruction: part 1
}

\section{Tsoumpas, Charalampos; Jørgensen, Jakob Sauer; Kolbitsch, Christoph; Thielemans, Kris}

Published in:

Philosophical Transactions of the Royal Society A: Mathematical, Physical and Engineering Sciences

Link to article, DOI:

10.1098/rsta.2020.0189

Publication date:

2021

Document Version

Publisher's PDF, also known as Version of record

Link back to DTU Orbit

Citation (APA):

Tsoumpas, C., Jørgensen, J. S., Kolbitsch, C., \& Thielemans, K. (2021). Synergistic tomographic image reconstruction: part 1. Philosophical Transactions of the Royal Society A: Mathematical, Physical and Engineering Sciences, 379(2200), 5. [20200189]. https://doi.org/10.1098/rsta.2020.0189

\section{General rights}

Copyright and moral rights for the publications made accessible in the public portal are retained by the authors and/or other copyright owners and it is a condition of accessing publications that users recognise and abide by the legal requirements associated with these rights.

- Users may download and print one copy of any publication from the public portal for the purpose of private study or research.

- You may not further distribute the material or use it for any profit-making activity or commercial gain

- You may freely distribute the URL identifying the publication in the public portal 


\section{PHILOSOPHICAL TRANSACTIONS A}

royalsocietypublishing.org/journal/rsta

\section{Introdution}
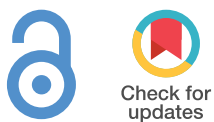

Cite this article: Tsoumpas C, Jørgensen J, Kolbitsch C, Thielemans K. 2021 Synergistic tomographic image reconstruction: part 1. Phil. Trans. R. Soc. A 379: 20200189. https://doi.org/10.1098/rsta.2020.0189

Accepted: 10 March 2021

One contribution of 9 to a theme issue 'Synergistic tomographic image reconstruction: part 1'.

\section{Subject Areas:}

medical physics, computational mathematics, biomedical engineering, image processing, computer modelling and simulation, artificial intelligence

\section{Keywords:}

positron emission tomography, computed tomography, magnetic resonance imaging, electrical impedance tomography, tomography, imaging

\section{Author for correspondence:}

Charalampos Tsoumpas

e-mail: tsoumpas@ieee.org

\section{Synergistic tomographic image reconstruction: part 1}

Charalampos Tsoumpas ${ }^{1,2,3}$, Jakob Sauer

Jørgensen ${ }^{4,5}$, Christoph Kolbitsch ${ }^{6,7}$ and

\section{Kris Thielemans ${ }^{8}$}

${ }^{1}$ Biomedical Imaging Science Department, University of Leeds, Leeds, West Yorkshire, UK

${ }^{2}$ Biomedical Engineering and Imaging Institute, Icahn School of Medicine at Mount Sinai, New York, NY, USA

${ }^{3}$ Invicro, London, UK

${ }^{4}$ Department of Applied Mathematics and Computer Science, Technical University of Denmark, Kongens Lyngby, Denmark ${ }^{5}$ Department of Mathematics, The University of Manchester, Manchester, UK

${ }^{6}$ Physikalisch-Technische Bundesanstalt, Braunschweig and Berlin, Germany

${ }^{7}$ School of Biomedical Engineering and Imaging Sciences, King's College London, London, UK

${ }^{8}$ Institute of Nuclear Medicine, University College London, London, UK

\section{CT, 0000-0002-4971-2477; JSJ, 0000-0001-9114-754X; CK, 0000-0002-4355-8368; KT, 0000-0002-5514-199X}

This special issue focuses on synergistic tomographic image reconstruction in a range of contributions in multiple disciplines and various application areas. The topic of image reconstruction covers substantial inverse problems (Mathematics) which are tackled with various methods including statistical approaches (e.g. Bayesian methods, Monte Carlo) and computational approaches (e.g. machine learning, computational modelling, simulations). The issue is separated in two volumes. This volume focuses mainly on algorithms and methods. Some of the articles will demonstrate their utility on realworld challenges, either medical applications (e.g. cardiovascular diseases, proton therapy planning)

(C) 2021 The Authors. Published by the Royal Society under the terms of the Creative Commons Attribution License http://creativecommons.org/licenses/ by $/ 4.0 /$, which permits unrestricted use, provided the original author and source are credited. 
or applications in material sciences (e.g. material decomposition and characterization). One of the desired outcomes of the special issue is to bring together different scientific communities which do not usually interact as they do not share the same platforms (such as journals and conferences).

This article is part of the theme issue 'Synergistic tomographic image reconstruction: part $1^{\prime}$.

\section{Introduction}

Traditionally tomographic image reconstruction has focused on estimating two-dimensional or three-dimensional images from a single modality dataset and acquisition. In recent years, however, there have been significant developments in hardware and systems that allow extracting multi-parametric images from multiple datasets. Examples include multi-spectral X-ray Computed Tomography (CT), multi-sequence Magnetic Resonance Imaging (MRI), multimodality imaging systems such as Positron Emission Tomography (PET) combined with MRI or $\mathrm{CT}$, acquisitions from multiple time-points (i.e. time-lapse tomography) and imaging multiple radiotracers at the same or in separate sessions. In such cases, it is common practice to reconstruct several images independently, each corresponding to single or multiple parameters extracted from a single or multiple acquisitions. However, it is often advantageous, although challenging, to combine information to jointly reconstruct multiple images, while exploiting common features between these images. Such 'synergistic image reconstruction' methods are the topic of this special issue.

The special issue grew from the Symposium on Synergistic Image Reconstruction held in the autumn of 2019 in Chester (UK) which brought together many of the experts in the field (more information can be found in the corresponding website of the symposium: https:/ /www. ccpsynerbi.ac.uk/symposium2019). The issue aims to create a collection of articles at the forefront of this subject from different research fields and application areas, including medical, biological and industrial imaging, to disseminate novel ideas, and present state-of-the-art research that will help accelerate new concepts and developments across all these different imaging fields.

The issue includes scientific articles across different application areas with one common theme: reconstruction of images by combining various different types of information. Several different approaches are used to solve the presented Inverse Problems. These include Bayesian methods, machine learning and deep learning algorithms as well as mathematical, physical, computational and when possible physiological modelling. The applications span from material sciences to scanning patients with advanced imaging such as for example integrated PET-MRI scanners. The special issue is separated in two different parts. The first issue (Part 1) includes three review articles and five research investigations. In both issues, there are articles which demonstrate the important utility of ideas when applied to answer application-specific questions. The imaging technologies featured in the first part of the special issue include but are not limited to spectral X-ray CT, MRI, PET-MRI, PET/CT, Electrical Impedance Tomography (EIT) and Quasi-Static Elasticity Imaging (QSEI). Many of these techniques have been developed only recently. For example, spectral CT has only recently appeared as a commercial clinical product and PET-MRI has been available for about 10 years as a combined clinical imaging machine, but there is still limited synergy between the two imaging techniques.

\section{Contributions in this issue}

The issue commences with a review by Arridge et al. [1] on synergistic image reconstruction methods which attempts to give the reader an update on the most recent published advances, while drawing similarities between the developments across different research fields. 


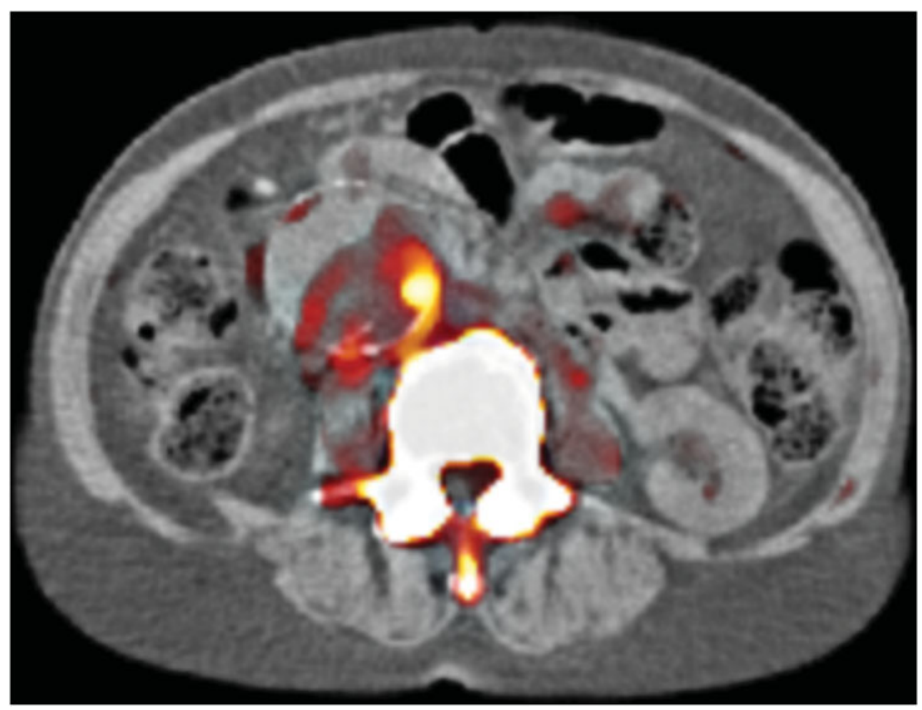

Figure 1. $\left[{ }^{18} \mathrm{~F}\right] \mathrm{NaF} \mathrm{PET}$ image of a patient with abdominal aortic aneurysm as reconstructed with a synergistic algorithm using the CT image for guidance [7]. (Online version in colour.)

MRI is a versatile imaging modality providing a range of different diagnostic information. Recently, quantitative MRI (qMRI), which produces maps of (bio)-physical parameters, has gained interest. These parameter maps are independent of any acquisition protocols or hardware issues and help reduce variability across different scans, devices, sites and patients leading to higher reproducibility. Clinically interesting parameters range from $\mathrm{T} 1, \mathrm{~T} 2$ and $\mathrm{T} 2 *$ relaxation times to measuring blood flow velocities. The special issue continues with two reviews on qMRI.

Qi et al. give an overview of synergistic image reconstruction for multi-contrast cardiac parameter mapping. The data redundancy for mapping of multiple parameters can be used using e.g. sparsity or low-rank constraints during image reconstruction. This allows for higher undersampling factors (i.e. faster scans) while maintaining high image quality. One of the challenges is long reconstruction times. Deep learning-based parameter mapping is a promising alternative which ensures accurate parameter estimation in a clinically feasible time-frame [2].

The above review focuses on approaches where qMRI is carried out in two steps: in the first step, qualitative images are reconstructed from the acquired k-space data and in the second step, a model is used to obtain the quantitative parameters from these images. Model-based image reconstruction produces the parameters directly from the acquired k-space data without any intermediate image reconstruction. This leads to a non-linear reconstruction problem which is challenging to solve, but enables efficient qMRI. Wang et al. review model-based image reconstruction for different quantitative parameters and discuss several strategies to solve such problems [3].

PET/CT scanning allows using CT images for guided PET reconstruction methods, where care needs to be taken with PET-only features. Deidda et al. demonstrate the usefulness of a synergistic image reconstruction method on a dataset of 72 human PET/CT scans primarily of patients with abdominal aortic aneurysms by using the kernelized expectation maximization (KEM) [4] and the hybrid KEM [5] algorithms. These two algorithms are compared with the conventionally used iterative image reconstruction algorithm: ordered subsets maximum likelihood expectation maximization (OSEM) [6]. The results illustrate that both KEM approaches can recover better the uptake of radioactivity in the aneurysms and potentially improve the diagnostic accuracy by up to $22 \%$ when compared to OSEM post-reconstructed filtered images [7] (figure 1). 
Machine learning is becoming more and more important for medical image reconstruction. Lv et al. carry out a comparison of MRI image reconstruction approaches based on four different generative adversarial networks (GAN). The study was carried out on brain, knee and liver datasets which were retrospectively undersampled by factors of 2, 4 and 6 . RefineGAN, which is the network achieving the overall highest image scores, showed high performance for all anatomies and all undersampling factors [8]. Future studies on multi-coil k-space data and including pathologies in the images are necessary.

Possible synergies exist not only between different types of reconstructed images. In simultaneous PET-MRI, both modalities are affected by the same physiological motion caused by breathing or the beating of the heart. Mayer et al. show that using images from both modalities for motion estimation through synergistic PET-MRI image registration allows for more accurate cardiac and respiratory motion estimation. This can improve the quality of $\left[{ }^{18} \mathrm{~F}\right] \mathrm{NaF}$ PET images of coronary plaques [9].

Within X-ray CT the development of new energy-discriminating detectors has motivated a tremendous research effort known as spectral CT with the possibility for improved material characterization and decomposition by synergistic exploitation of tomographic data measured over a range of $\mathrm{X}$-ray energies. Perelli and Andersen propose a regularization method for multi-material decomposition in spectral CT [10]. This is based on denoising using a datadriven regularizer expressing complex prior structure. An efficient second-order optimization algorithm based on non-uniform random subsampling of the Hessian matrix is proposed to solve the problem. Experiments with synthetic and real datasets from a physical cylinder phantom demonstrate that the proposed method is capable of computing a reliable material decomposition producing comparable results with an existing method.

Hauptmann and Smyl present a synergistic approach for combining EIT and QSEI [11]. In EIT, an internal conductivity distribution is reconstructed from voltage measurements taken at the object boundary. In QSEI, the elastic modulus distribution is reconstructed from a displacement field measured within the object domain rather than at the boundary, and in this sense QSEI forms a complementary modality to EIT. Both EIT and QSEI have potential applications for example in medical imaging, however both are highly ill-posed, non-linear inverse problems. The authors propose a synergistic reconstruction method employing joint total variation to enforce structural similarity in particular common edges for the two modalities. Using simulation experiments for a case study of localization of cracks, they demonstrate improvements in image quality with the synergistic approach compared to single-modality TV-regularized reconstruction.

The contributions in this first part of the special issue provide an overview of the different scientific fields as well as important advances. We anticipate further exciting advances in Part 2, as well as several software-oriented papers. The latter describe open source software packages enabling researchers to explore synergistic image reconstruction methods. Taken together, we believe that this special issue highlights synergistic image reconstruction as a fertile area with scope for future research and ultimately fruitful application domains.

Data accessibility. This article has no additional data.

Authors' contributions. C.T. drafted the manuscript; J.S.J., C.K. and K.T. wrote sections and revised the manuscript; and all authors read and approved the manuscript.

Competing interests. C.T. serves as an executive member of the advisory board of Positrigo AG, Zürich, Switzerland. K.T. is the Director of Algorithms and Software Consulting Ltd, London, UK. The other authors report no conflicts of interest.

Funding. This work was funded by the UK EPSRC grants no. 'Computational Collaborative Project in Synergistic PET/MR Reconstruction' (CCP PETMR) EP/M022587/1 and its associated Software Flagship project EP/P022200/1; the 'Computational Collaborative Project in Synergistic Reconstruction for Biomedical Imaging' (CCP SyneRBI) EP/T026693/1; 'A Reconstruction Toolkit for Multichannel CT' EP/P02226X/1 and 'Collaborative Computational Project in tomographic imaging' (CCPi) EP/M022498/1 and EP/T026677/1. C.T. is sponsored by a Royal Society Industry Fellowship (IF170011). J.S.J. was partially supported by The Villum Foundation (grant no. 25893). 
Acknowledgements. We wish to thank Ms Alice Power who, as the commissioning editor of the journal, has provided great support in the timely and appropriate completion of the special issue. Furthermore, we wish to thank all authors and reviewers of the articles for their work that made this special issue possible even during a pandemic.

\section{References}

1. Arridge SR, Ehrhardt MJ, Thielemans K. 2021 (An overview of) Synergistic reconstruction for multimodality/multichannel imaging methods. Phil. Trans. R. Soc. A 379, 20200205. (doi:10.1098/rsta.2020.0205)

2. Qi H, Cruz G, Botnar R, Prieto C. 2021 Synergistic multi-contrast cardiac magnetic resonance image reconstruction. Phil. Trans. R. Soc. A 379, 20200197. (doi:10.1098/rsta.2020.0197)

3. Wang X, Tan Z, Scholand N, Roeloffs V, Uecker M. 2021 Physics-based reconstruction methods for magnetic resonance imaging. Phil. Trans. R. Soc. A 379, 20200196. (doi:10.1098/ rsta.2020.0196)

4. Wang G, Qi J. 2015 PET image reconstruction using kernel method. IEEE Trans. Med. Imaging 34, 61-71. (doi:10.1109/TMI.2014.2343916)

5. Deidda D, Karakatsanis NA, Robson PM, Tsai YJ, Efthimiou N, Thielemans K, Fayad ZA, Aykroyd RG, Tsoumpas C. 2019 Hybrid PET-MR list-mode kernelized expectation maximization reconstruction. Inverse Prob. 35, 044001. (doi:10.1088/1361-6420/ab013f)

6. Hudson H, Larkin R. 1994 Accelerated image reconstruction using ordered subsets of projection data. IEEE Trans. Med. Imaging 13, 601-609. (doi:10.1109/42.363108)

7. Deidda D et al. 2021 Improved identification of abdominal aortic aneurysm using the Kernelized Expectation Maximization algorithm. Phil. Trans. R. Soc. A 379, 20200201. (doi:10.1098/rsta.2020.0201)

8. Lv J, Zhu J, Yang G. 2021 Which GAN? A comparative study of generative adversarial network-based fast MRI reconstruction. Phil. Trans. R. Soc. A 379, 20200203. (doi:10.1098/ rsta.2020.0203)

9. Mayer J, Jin Y, Wurster T-H, Makowski MR, Kolbitsch C. 2021 Evaluation of synergistic image registration for motion-corrected coronary NaF-PET-MR. Phil. Trans. R. Soc. A 379, 20200202. (doi:10.1098/rsta.2020.0202)

10. Perelli A, Andersen MS. 2021 Regularization by denoising sub-sampled Newton method for spectral CT multi-material decomposition. Phil. Trans. R. Soc. A 379, 20200191. (doi:10.1098/ rsta.2020.0191)

11. Hauptmann A, Smyl D. 2021 Fusing electrical and elasticity imaging. Phil. Trans. R. Soc. A 379, 20200194. (doi:10.1098/rsta.2020.0194) 